\title{
Reverberation Mapping: Masses and Distance and Size, Oh My!
}

\section{K. D. Denney ${ }^{* \dagger}$}

The Ohio State University, Department of Astronomy, 140 W. 18th Avenue, Columbus, OH 43221, USA

E-mail: denneydastronomy .ohio-state.edu

\begin{abstract}
I review the technique of reverberation mapping and describe its use in the direct, dynamical mass measurement of actively accreting supermassive black holes located at the centers of active galactic nuclei (AGN). I discuss continued challenges to this method that current and planned observations are working to overcome. I also present some additional applications of the reverberation mapping measurements, discussing how reverberation time delays used to probe the size of the broad line-emitting region not only allow for these direct mass measurements, but also, through empirically calibrated scaling relationships, provide a method for indirect mass estimates of black holes in large samples of distant quasars. Furthermore, these broad-line region size measurements also have the potential to turn AGN into cosmic distance probes, as the measured size of this region scales with intrinsic AGN luminosity. With regard to these applications, I address some of the continuing sources of systematic uncertainties with which I am currently concerned, as well as work that is being done in an attempt to understand and mitigate these systematics.
\end{abstract}

Frank N. Bash Symposium 2013: New Horizons in Astronomy (BASH 2013)

October 6-8, 2013

Austin, Texas

\footnotetext{
${ }^{*}$ Speaker.

$\dagger$ I would like to acknowledge my collaborators and other collaborations who are performing reverberation mapping and related projects, without whom this review and the exciting state of this field would not have been possible. I would also specifically like to thank C. J. Grier, A. Pancoast, and D. Park, for allowing me to include figures from their work as a means to highlight ongoing advances in reverberation mapping. KDD would also like to acknowledge support through NSF AAPF grant AST-1302093.
} 


\section{Introduction}

One of the fundamental goals in astronomy is to be able to explain our place in the Cosmos. In particular, we want to understand the evolution of the Universe and what events and processes have taken place to result in the locally observable universe at the present day. The first step toward attaining this goal is to map out and catalogue the observable universe and to take stock of the demographics by measuring physical attributes of its contents. Finding ways to measure attributes such as the mass, distance to, and size of astronomical objects can be likened to hunting 'big game' - difficult but ultimately rewarding (if you like that kind of thing). If you caught the hidden reference to lions and tigers and bears in the title of this contribution, this is because I am suggesting that the technique of reverberation mapping is a useful and powerful tool (or weapon) for hunting this big game. In this contribution, I will describe how reverberation mapping is able to make these measurements of mass (of supermassive black holes; BHs), distance (to these BHs and their host galaxies), and size (of the gas distribution surrounding the $\mathrm{BH}$ ), and how making these measurements contributes to our understanding of some of the large scale, fundamental ambitions in astronomy research today.

\section{Black Holes and Their Place in the Universe}

Our current understanding of the large scale structure and evolution of the universe has evolved and strengthened through both observation and theory. For example, redshift surveys such as the 2dF Galaxy Redshift Survey [1] and the Sloan Digital Sky Survey [e.g., 2] have provided a map of the local universe showing the interesting spatial distribution of galaxies that has become coined "the cosmic web" [3]. Cosmological N-body simulations, such as the Millennium Simulation [4] are able to generally reproduce the observable universe by utilizing a $\Lambda \mathrm{CDM}$ cosmology, i.e., one containing both cold dark matter (CDM) and dark energy in the form of a cosmological constant, $\Lambda$. By comparing simulations such as these to the observations, the basic paradigm for the creation of large scale cosmic structure is by hierarchical growth through gravitational instabilities from density fluctuations present in the early universe.

Within this paradigm, several pieces of evidence suggest the existence and importance of supermassive $\left(\gtrsim 10^{6} M_{\odot}\right)$ black holes in this structure growth and evolution, including, but not limited to, (1) the existence of Active Galactic Nuclei (AGN) or quasars - BHs growing in the centers of galaxies that, in the process, emit copious amounts of radiation, (2) the presence of a quiescent (not significantly growing) $\mathrm{BH}$ in the center of our own as well as other nearby massive galaxies, and (3) observed correlations between the mass of a central BH and its host galaxy properties in both quiescent (here, galaxies with BHs that are not active) and active (those with actively accreting BHs) galaxies, which may be an indication of co- (even if not causal) evolution $[5 ; 6 ; 7 ; 8 ; 9 ; 10 ; 11 ; 12$, see also McConnell, these proceedings]. One implication behind these observations is that tracing the masses and growth of BHs over cosmic time may add significant pieces to the overall picture of how the universe and the galaxies within it have evolved since early times. Tracing this structure growth also constrains the underlying cosmology, which impacts this evolution. 


\section{Direct Measurements of the Mass of Black Holes}

All direct mass measurement methods must somehow probe the size scale where the motions of matter are dominated by the gravitational potential of the $\mathrm{BH}$ rather than that of the global galaxy potential. Within this "sphere of influence", the dynamics of the matter itself must be measured or inferred, so that a virial mass can be determined from a combination of the velocity of the material probed, $V$, and the distance of this material from the $\mathrm{BH}, R$, such that $M_{\text {virial }} \sim R V^{2} / G$, where $G$ is the gravitational constant. In our own galaxy, the orbits of individual stars around Sagittarius $A^{*}$ can be determined, but this is not possible for even the most nearby extragalactic $\mathrm{BH}$. For relatively nearby galaxies (currently within $\sim 150 \mathrm{Mpc}$, though ultimately dependent on the $\mathrm{BH}$ mass probed and current telescope technology), while individual stars cannot be resolved, populations of stars and/or distributions of gas with this sphere of influence can still be spatially resolved. In these systems, the spatially resolved stellar or gas dynamics within the central regions of the galaxy are used to derive the mass of the central BH, though the results are still somewhat model dependent. See the contribution to these proceedings by Nicholas McConnell for more information on the details and results for these types of dynamical black hole mass measurements.

Another direct mass measurement method I will mention here briefly for completeness, but that is generally outside the scope of this review, is the method of using $\mathrm{H}_{2} \mathrm{O}$ mega-masers observed in the dusty disks, or possibly "tori", surrounding and obscuring Seyfert 2, or non-broad lineemitting, AGN. In these systems, a chance alignment of the maser emission with our line-of-sight provides a means by which radio interferometry observations can probe both the spatially resolved location and velocity distribution of this gas. As this emission arises from gas well within the gravitational sphere of influence of the $\mathrm{BH}$, these measurements allow for the determination of the mass of the $\mathrm{BH}$. Megamasers provide the most precise and most accurate measurements of extragalactics BH masses, but unfortunately, such systems are relatively rare, and spatial resolution is still required, thereby limiting this method to the local universe [see, e.g., 13; 14; 15, for more details].

\subsection{Moving to the Time Domain: Reverberation Mapping}

Reverberation mapping [RM; 16; 17] is a method for measuring dynamical black hole masses in broad line, or Type 1, AGN that is currently the only dynamical method that is not distance dependent, i.e., it is applicable outside the local universe. This is because RM is not reliant upon spatial resolution of the gravitational sphere of influence of the $\mathrm{BH}$, but instead, works in the time domain. This method requires time resolution of brightness fluctuations observed in (i) the continuum emission from the BH's thermal accretion disk and (ii) the reprocessed broad line emission arising from gas photo-ionized by the continuum radiation and located very near the black hole (e.g., within sub-parsec scales). There is an observed time delay, or lag, $\tau$, between the variations in the continuum and reprocessed broad emission-line flux corresponding to the light travel time between these two regions. This reverberation lag thereby infers the radius of the broad line-emitting region (BLR), $R_{\mathrm{BLR}}=c \tau$, whose gas motions, $V$, are dominated by the gravity of the central $\mathrm{BH}$, and therefore manifest as line-of-sight Doppler broadening of the emission lines. The virial mass of the $\mathrm{BH}$ can then be determined from the equation above, modulo a scale factor, $f$, that is of order unity and accounts for the unknown BLR geometry and kinematics. 

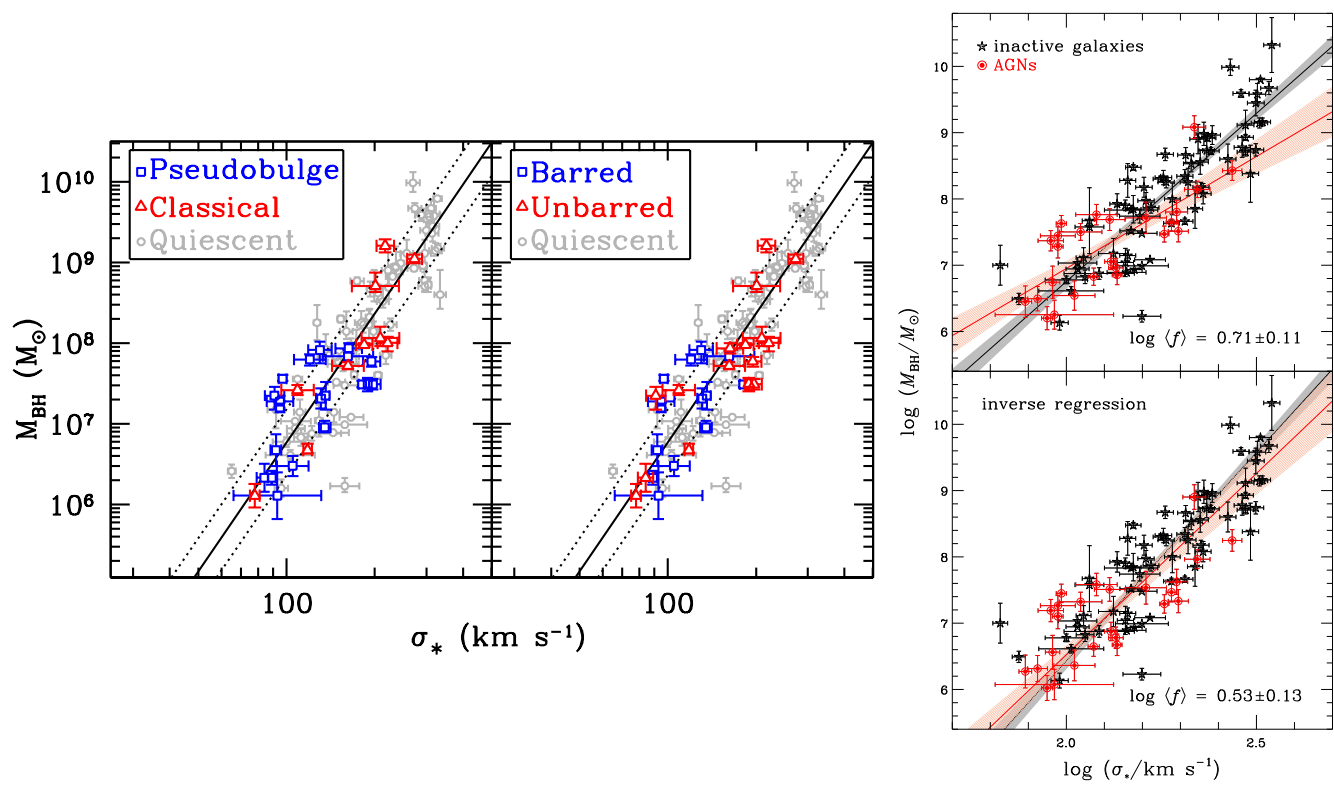

Figure 1: Left: Reproduced from Grier et al. [12]. The $M-\sigma_{*}$ relation for the updated AGN sample discussed by Grier et al. [12]. The gray points represent the quiescent galaxy sample, and the black solid and dotted lines are the best fit and intrinsic scatter of the quiescent sample. The colored points represent the AGN sample divided by bulge type (left panel) and the presence of a bar in the AGN host galaxy (right panel). All AGN black hole masses have been scaled by $\langle f\rangle=4.31$ as determined by Grier et al. Right: Reproduced from Park et al. [19]. The $M-\sigma_{*}$ relation for the AGN sample discussed by Park et al. for inactive (black) and active (red) galaxies. The two panels represent determination of $\langle f\rangle$ with a forward regression (top) or an inverse regression (bottom), using the F I TEXY estimator.

Reverberation-based masses, while still direct, dynamical mass measurements, currently suffer from a sample-wide systematic uncertainty because $f$ is typically unknown for individual objects (though see below). The RM-based mass scale is thus set by an ensemble zero-point calibration, $\langle f\rangle$, such that $M_{\mathrm{BH}-\mathrm{RM}}=\langle f\rangle M_{\text {virial }}$. This calibration is based on the assumption that active and quiescent galaxies follow the same $M-\sigma_{*}$ relationship [18] and has since been updated by Park et al. [19] and Grier et al. [12] as the sample of RM mass and corresponding bulge stellar velocity dispersion measurements has grown and been updated. Figure 1 highlights one important result of these latter two studies [see also, e.g., 20] that the $M-\sigma_{*}$ relationship, and therefore the determination of $\langle f\rangle$, is both sample and analysis dependent. This strongly suggests that determining an independent method by which to calibrate $\langle f\rangle$, or even more ideally, calculate $f$ for individual objects, is of significant value to precision $\mathrm{BH}$ mass measurements.

\subsection{Recent advances in Reverberation Mapping Analyses: Velocity Delay Maps and Broad Line Region Modeling}

Luckily, high level reverberation mapping analyses afforded by the more recent RM campaigns with very high quality data are making inroads into measuring $f$. These types of programs will or already are leading to RM-based mass measurements that are independent of other methods (i.e., do not depend on $\langle f\rangle)$. I show a couple examples of the products from these types of analyses in 

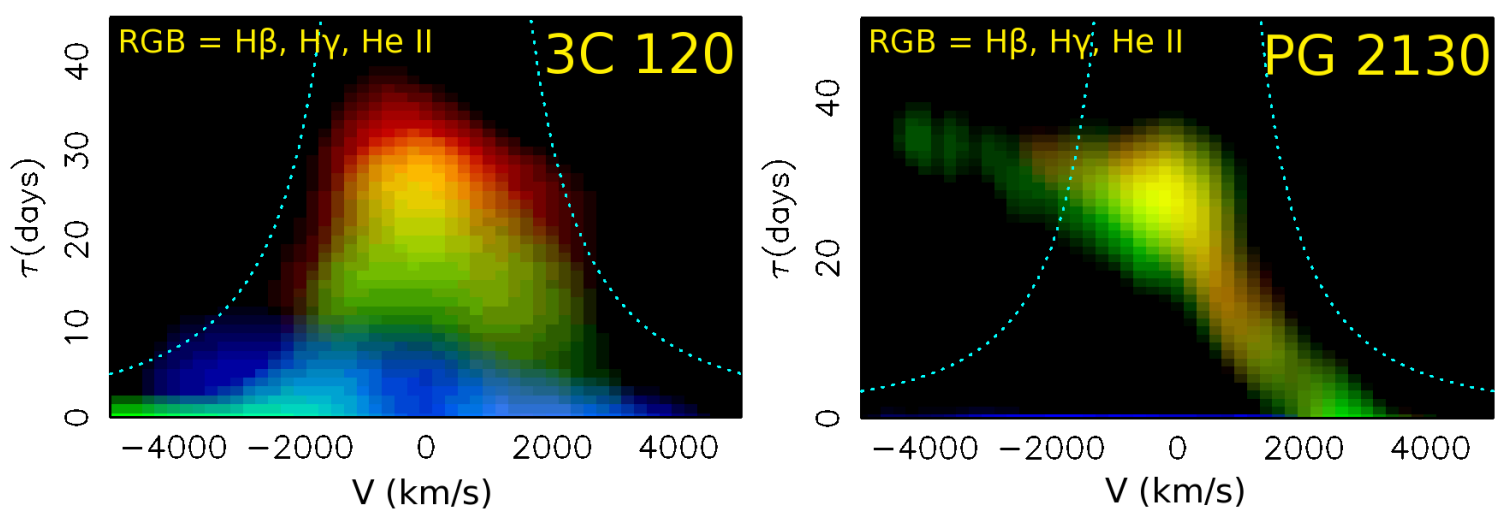

Figure 2: Reproduced from Grier et al. [25]. False-color velocity-delay maps for 3C 120 (left) and PG $2130+099$ (right) for reverberation responses from the $\mathrm{H} \beta$ (red), $\mathrm{H} \gamma$ (green), and $\mathrm{He}$ II $\lambda 4686$ (blue) emission lines. See Grier et al. [25] for details.

Figures 2 and 3. In particular, the information gained by the high quality and high time cadence of the observations gleaned from recent RM programs [e,g. 21;22;23;24] is allowing measurements of the differential reverberation lag in velocity space across the broad emission line. This allows for the determination of a solution to the 2-dimensional transfer function — in time delay and line of sight velocity space - otherwise known as a velocity delay map. Velocity delay maps, such as those shown in Figure 2 are solely based on data, but can then be interpreted through comparison to photoionization models of the BLR with various geometries and kinematics to estimate a value of $f$.

In a similar, though effectively opposite approach, the Bayesian analysis software of Pancoast et al. [26] uses dynamical models of the BLR with possible geometries in combination with the reverberation campaign spectra and light curves to derive the BH mass and constrain the BLR geometry (see Figure 3 ). This modeling process can also output the 2D velocity delay map as a product, but more importantly, this dynamical modeling results in an independent estimate of the black hole mass, i.e., one that does not depend on $\langle f\rangle$. Instead $f$ is derived by a comparison of this dynamical black hole mass estimate to $M_{\text {virial }}$ calculated through the traditional reverberation mapping analysis of the same data. Results from this method $[27 ; 26 ; 28]$ have been applied to half a dozen AGN so far.

Despite improvements in both stellar and gas dynamical methods and reverberation mapping analyses, it is still a major concern, as demonstrated by Figure 4, that nearly half of the objects for which the BH mass has been measured with multiple, independent methods, show that the results between methods are statistically inconsistent. We are still dealing with small number statistics, so one should not yet read too much into this trend. Nonetheless, for the sources that $d o$ agree, two (NGC 4151 and NGC 3227) are Type 1 AGN that have both reverberation mapping and galacticscale dynamical mass measurements, and the reverberation mapping measurement agrees to within the quoted uncertainties with one or both of the galaxy dynamics-based masses. In general, the typically assumed accuracy in RM-based masses comes from first assuming that there is no intrinsic scatter in the $M-\sigma_{*}$ relationship and that the observed scatter is then due to the different values of $f$ for individual sources. Given the current scatter in the AGN $M-\sigma_{*}$ relationship, this 

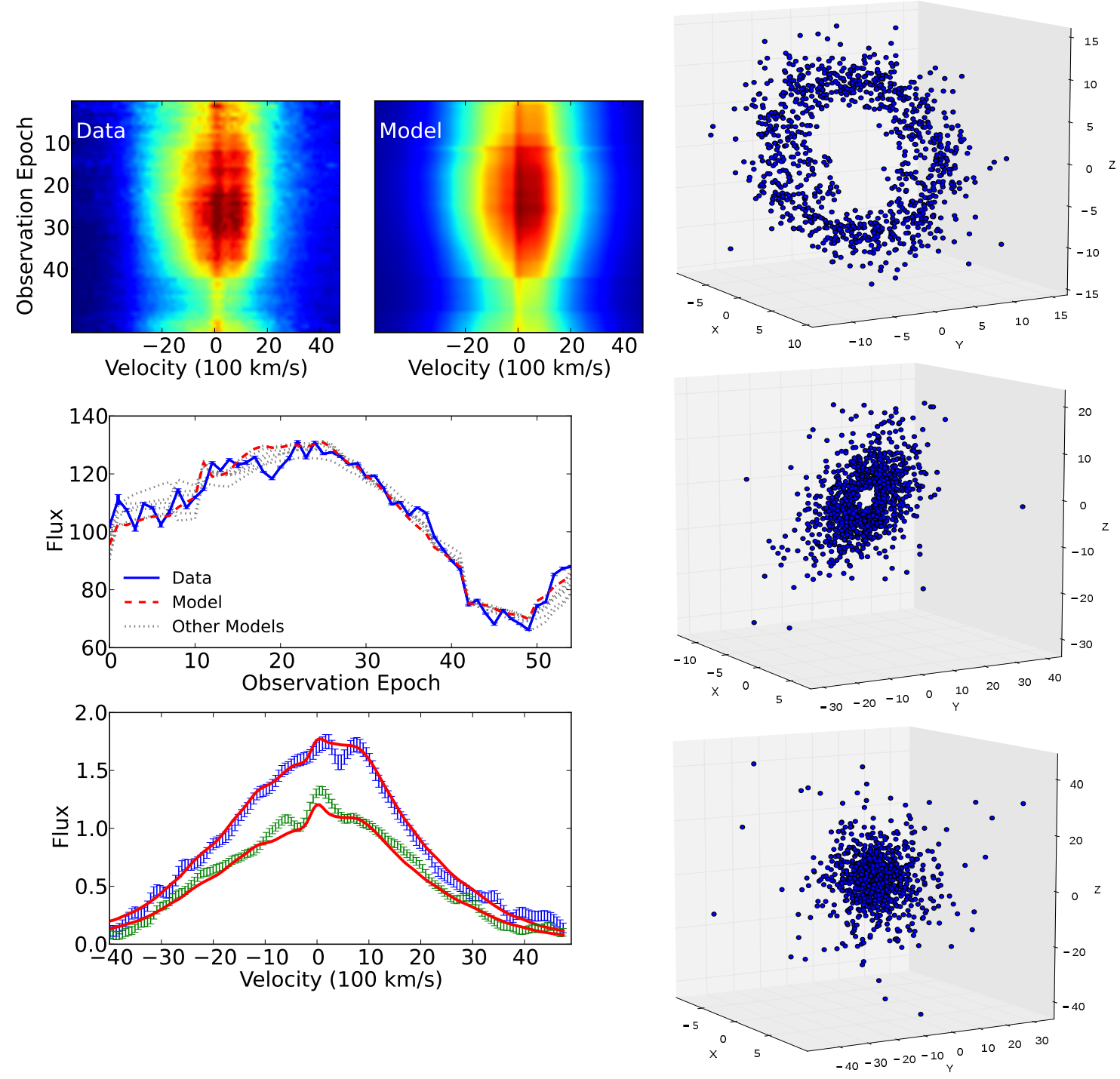

Figure 3: Reproduced from Pancoast et al. [26]. Left: $\mathrm{H} \beta$ line profile and light curve data and model fits from the LAMP 2011 reverberation campaign of Mrk 50. The top panels show the line profile flux intensity color-coded and stacked by epoch. The middle panel shows the $\mathrm{H} \beta$ light curve (blue) with the adopted model fit in red and alternate model fits as dotted gray curves. The bottom panel shows the model fit to the $\mathrm{H} \beta$ profile from two individual epochs. Right: BLR geometry for three different models. See Pancoast et al. [26] for details. 


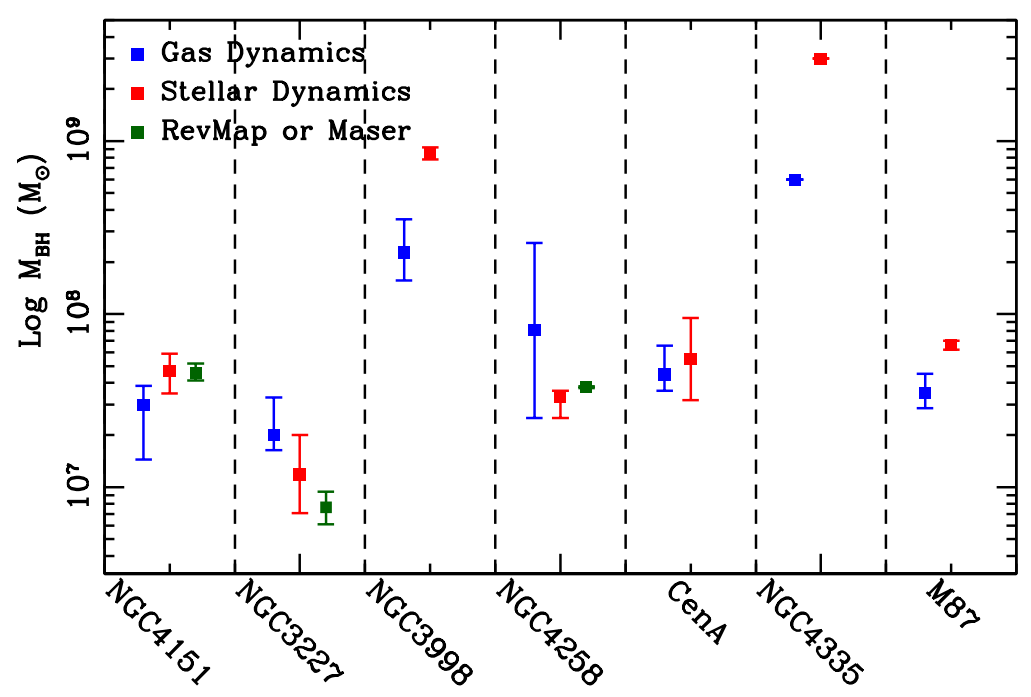

Figure 4: Comparison of black hole mass estimates between independent methods for the objects that have reliable results from multiple methods. Except for the stellar dynamical mass of NGC 4151, which was taken from Onken et al. 2014 (private communication; submitted for publication), all other gas and stellar dynamical masses (blue and red data points, respectively) are taken from Kormendy \& Ho [15], as is the adopted maser-based mass for NGC 4258 (green data point); see that work for references to individual studies. The reverberation mass measurements (also green points) of NGC 4151 and NGC 3227 are from Bentz et al. [29] and Denney et al. [22], respectively.

implies that any, single RM mass is systematically uncertain by $\sim 0.4$ dex. However, given that this assumption of zero intrinsic scatter is unlikely, the RM masses are more realistically good to better than a factor of $\sim 2$. Similarly, masses based on galactic stellar and gas dynamics are typically considered to be accurate to within a factor of two or so, where this systematic uncertainty is largely model-dependent [see 15, and McConnell, these proceedings]. This underscores the need for more consistency checks between methods to better constrain and understand systematics in all methods.

\section{Calibrating BH Mass Scaling Relationships with BLR Size Measurements}

RM has also confirmed a tight relationship between the BLR radius and AGN luminosity, the $R-L$ relation, expected as a consequence of the photoionization physics regulating the broad-line emission [30; 31]. The $R-L$ relation provides a simple, powerful means of estimating BH masses from a single spectrum for large samples of AGNs at any redshift: the AGN continuum luminosity is used as a proxy for the BLR radius and combined with the BLR velocity inferred from the broad emission-line width through the BH mass equation given above, producing a "single-epoch" (SE) mass estimate. Significant effort has been put into searching for and calibrating such a relationship with early RM results [e.g., 32; 33; 34], but it was not until host galaxy starlight contamination was removed from the measured AGN luminosity and early reverberation mapping results were revisited with new observations that the slope of the observed relationship became consistent with physical expectations and the scatter was significantly reduced $[35 ; 36 ; 37]$. Figure 5 shows the most up-to-date calibration of this relation that should be utilized for single-epoch black hole mass 


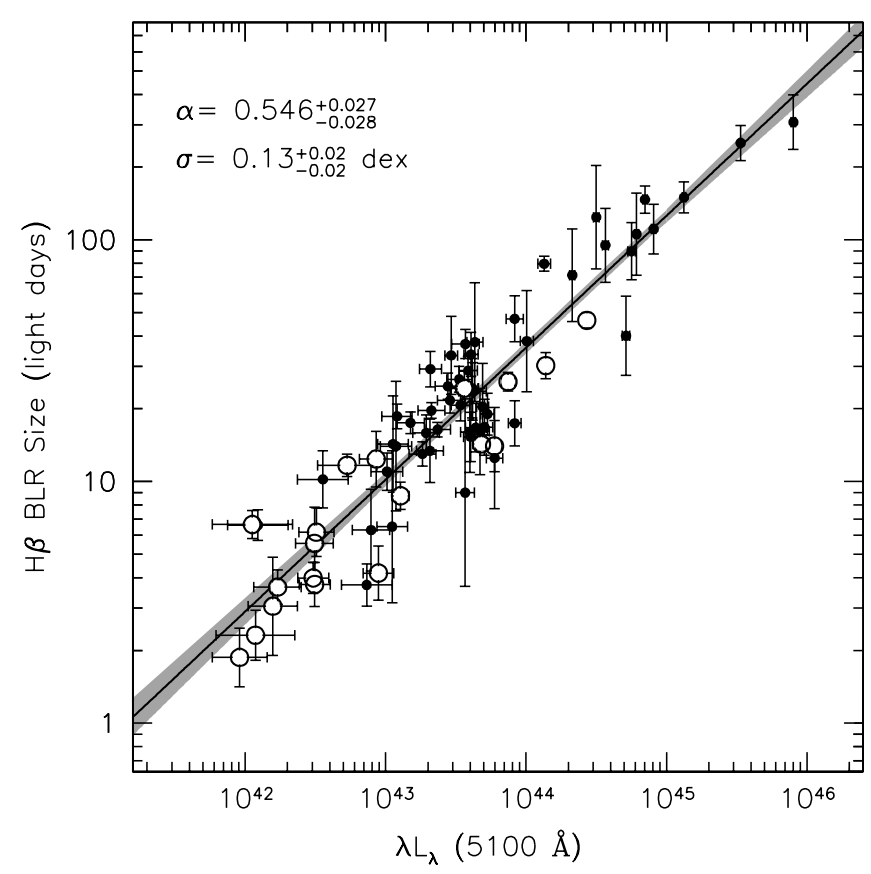

Figure 5: Reproduced from Bentz et al. [37]. H $\beta$ BLR radius measured from reverberation mapping versus the $5100 \AA$ AGN luminosity. The solid line is the best fit to the data, with the grayscale region demonstrating the range of slopes allowed by the uncertainties on the best fit. Open circles are the newest measurements included by Bentz et al. [37] for the first time, and all data points include error bars representing their associated uncertainties, though some may be smaller than the plot symbols. This version of the $R-L$ relationship does not include the full sample of 71 data points discussed by Bentz et al. Here, Mrk 142 is removed, the adopted lag for PG $2130+099$ is $31 \pm 4$ days, and a reddening correction of 0.26 dex for NGC 3227 has been applied. These adjustments do not appreciably alter the best fit slope but reduce the scatter from 0.19 dex to 0.13 dex [see 37 , for details].

estimates. Such a method is of supreme importance to studies of galaxy evolution and cosmic structure growth, as described above, as nearly all cosmologically distant black holes have had their masses estimated using this method. BH mass scaling relationships for estimating singleepoch masses based on these RM measurements are now available and widely used in the literature to estimate $\mathrm{BH}$ masses from the $\mathrm{H} \beta, \mathrm{MgII}$, CIV, and (sometimes) $\mathrm{H} \alpha$ broad emission lines.

\subsection{Velocity-width Biases in SE BH Mass Estimates}

Because of the prevalent use of these BH mass scaling relationships, understanding the source of and characterizing the limitations and systematic uncertainties in this method are imperative. A major difficulty is that there is currently little uniformity across studies in what observables and scaling relationships are used to derive the mass. Figure 6, originally presented by Park et al. [38] draws attention to the latter issue. Here, all SE BH masses are estimated using the full width at half maximum (FWHM) line width characterization of $\mathrm{H} \beta$, MgII, and CIV. However, the black points represent measurements using scaling relationships that follow a strict virial relation in the velocity, i.e., $M \propto V^{2}$, while the red points show the results using alternate calibrations of MgII and CIV based masses that relax this requirement, and instead, explicitly fit for the exponent of the 


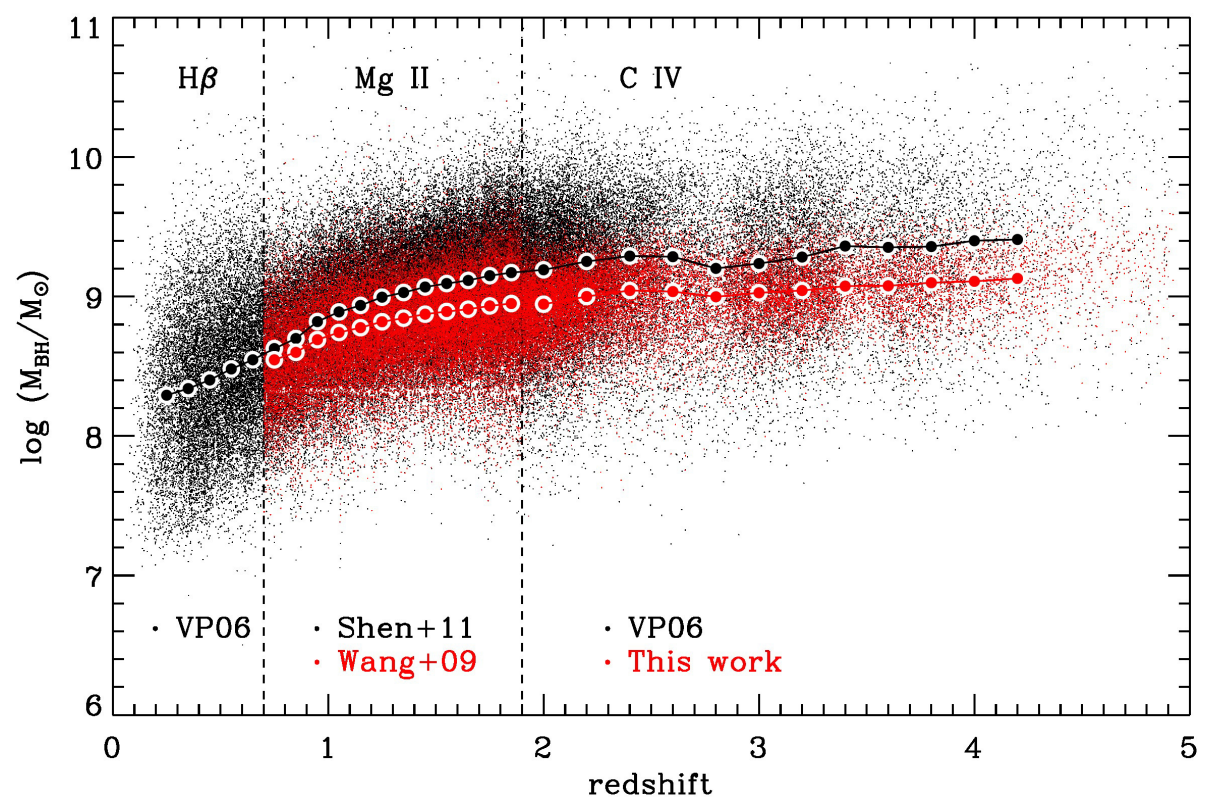

Figure 6: From Park et al. [38]. Comparison of FWHM-based BH masses as a function of redshift between different BH scaling relationship calibrations. The FWHM and luminosity measurements for these $\sim 100,000$ QSOs are taken from the SDSS DR7 quasar catalog [42] to calculate the black hole mass using different estimators. The emission line used and corresponding BH mass estimator reference are indicated in upper and bottom parts, respectively, of each redshift range separated by vertical dashed lines. Small dots represent individual mass estimates, while the large filled circles indicate the median values in each redshift bin, with $\Delta z=0.1$ for $z \leqslant 1.9$ and $\Delta z=0.2$ for $z>1.9$.

velocity dependence as a means to mitigate apparent systematic biases in the masses propagated from biases in the FWHM measurements [cf. 39; 40; 41; 38]. The average offset values of the red filled circles from black filled circles are 0.17 dex for MgII and 0.25 dex for CIV.

A significant contribution to these differences in SE mass scaling relationship calibrations is that there can be significant systematic differences present in SE masses depending on the approach adopted for characterizing the BLR gas velocity dispersion via the broad emission-line width. This is particularly true for the CIV emission line. Many studies contend that there are unacceptably large uncertainties and systematic biases in CIV-based masses compared with $\mathrm{H} \beta$ [e.g., 43; 44; 45]. The main point of contention for CIV is that the line profile is often observed to be either blueward asymmetric or the peak is blue-shifted with respect to the systemic redshift, or both, which can indicate non-virial, particularly outflowing, motions of the gas. This is a concern for the $\mathrm{BH}$ mass measurements if these gas motions are not, in fact, dominated by the gravity of the $\mathrm{BH}$. Nevertheless, because CIV is observed in the optical for $z \gtrsim 1.5$, it is the most applicable line for studying the high redshift universe, where the most dramatic evolution is occurring, and therefore attractive for $\mathrm{BH}$ mass and $\mathrm{BH}$ growth studies.

Recent results by Denney [41] shed light on the possible cause for at least a large fraction of the systematic biases present in CIV-based single-epoch BH masses, compared to those based on $\mathrm{H} \beta$. Analysis of past RM results of CIV show the presence of a non-variable component in the CIV profile. This is demonstrated in Figure 7 by the comparison of the CIV profile in the reverberation 

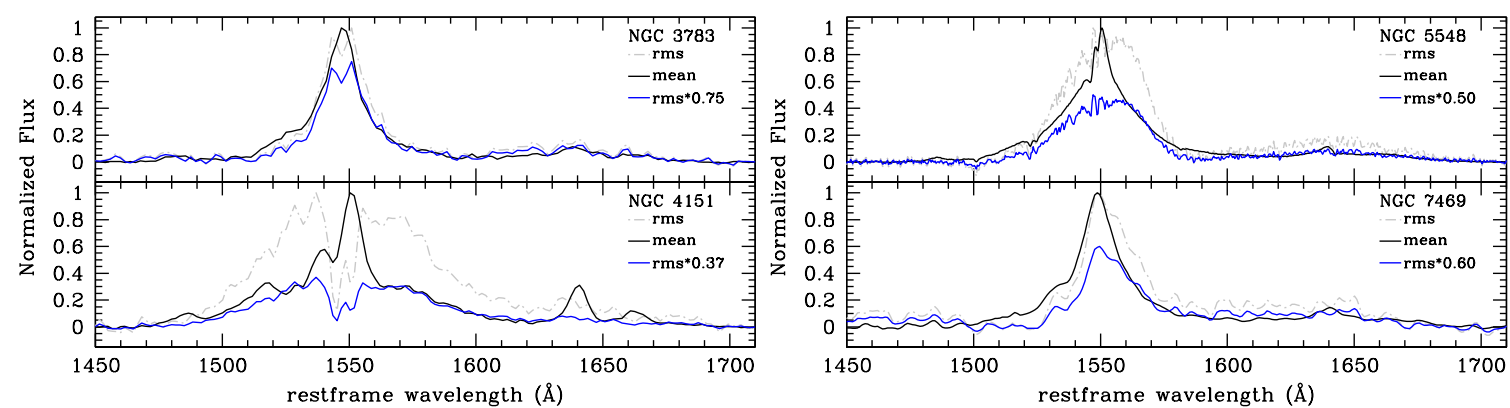

Figure 7: Examples of continuum-subtracted mean (black) and rms (gray dot-dashed) spectra from the C IV RM sample that have been normalized to the C IV peak flux. The blue curves show the same rms spectra scaled by an arbitrary factor to approximately match the red wing flux between each rms and mean spectrum. Adapted from Denney [41].

campaign mean spectrum - that averaged from all individual spectra taken during the campaign - and the rms spectrum - the rms residual flux with respect to the mean over the duration of the campaign. The rms spectrum probes the actual reverberating BLR gas, i.e., that which is variable on the reverberation timescales. On the other hand, in the mean or a single-epoch spectrum, we are observing all the emission along the line-of-sight, not necessarily only the reverberating BLR emission. Through this comparison, Denney [41] shows that there is a distinctly "non-variable" component of the CIV emission that, while largely concentrated in the low-velocity core of the profile, is (i) broader than the narrow forbidden line emission from the narrow line region (NLR), (ii) not easily distinguished from the broad component, as common for the non-variable NLR emission component of, e.g., $\mathrm{H} \beta$, and (iii) generally non-Gaussian, often having instead a profile with a blueward asymmetric wing that also seems to differ with the overall profile of CIV - the more blueshifted the line peak is from systemic, the more asymmetric the profile and stronger the blue wing. The consequence of the presence of this non-variable component is a bias in CIV-based single-epoch masses due to contamination from the non-variable component to the measurement of the line width relevant for probing the "reverberating" BLR gas velocity dispersion (that expected at the radius inferred from the $R-L$ relation).

CIV based masses measured directly from RM experiments are not biased in this way because the BLR velocities are measured from the line profile in the rms spectrum and therefore probe the velocities of only the reverberating gas, whose radius is measured from the time delay. For objects with RM results for multiple lines, the masses derived from the different lines are mutually consistent. While reassuring from the physical standpoint of the reliability of CIV as a virial mass indicator, in general, this does not specifically solve the problem of single-epoch masses, which are the most widely applicable. Furthermore, it is, unfortunately, non-trivial to simply subtract this CIV non-variable component as is done for the NLR component of $\mathrm{H} \beta$ because its origin is not yet well-understood, it cannot be modeled as a Gaussian, nor is its width consistent with that of other forbidden NLR emission lines. Consequently, FWHM-based CIV masses are susceptible to the largest biases, up to an order of magnitude in the mass, because of the dependence of the FWHM on the line peak, which is the most contaminated. This explains why past studies, which were all using CIV masses based on the FWHM, found such poor agreement with $\mathrm{H} \beta$-based masses. 


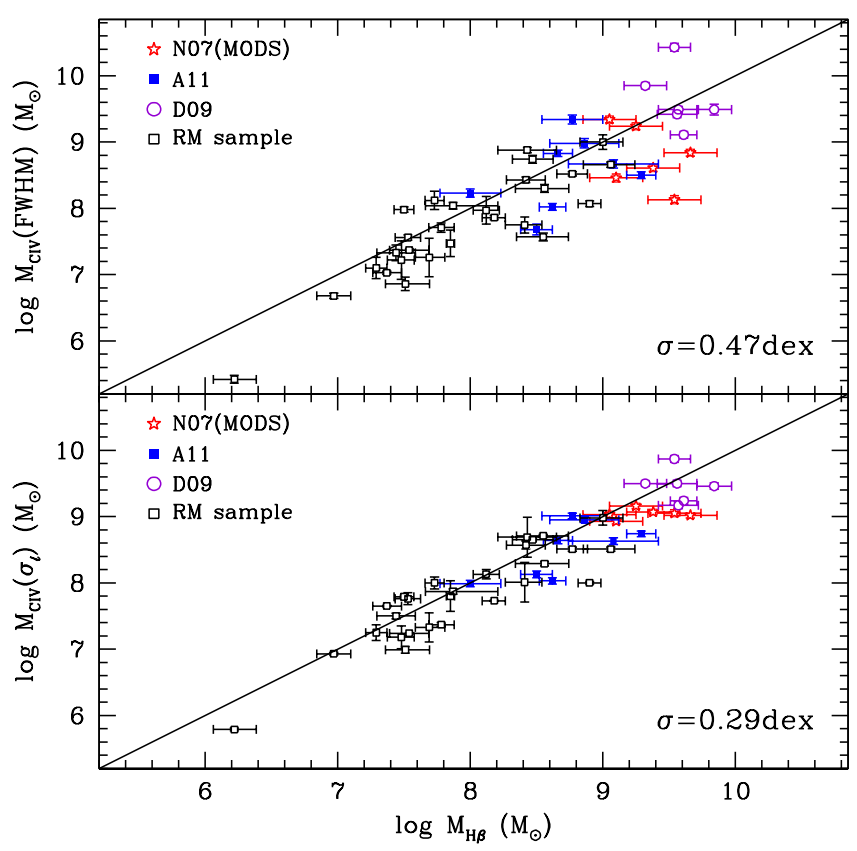

Figure 8: Adapted from Denney et al. [46]. Comparison between H $\beta$-based masses and C IV masses estimated from a sample with high quality spectra. The top (bottom) panel shows C IV masses based on the $\operatorname{FWHM}\left(\sigma_{l}\right)$. The solid line shows where the $\mathrm{CIV}$ and $\mathrm{H} \beta$ masses are equal. The scatter, $\sigma$, quantified as the standard deviation about the mean of the sample of $\mathrm{C}$ IV-to- $\mathrm{H} \beta$ mass residuals, $\log M(\mathrm{CIV})-\log M(\mathrm{H} \beta)$, is shown in the bottom right of each panel. Different data point colors represent objects from different samples; see Denney et al. [46] for details.

Instead, the most straightforward way, currently, to mitigate this bias is to use the line dispersion, $\sigma_{l}$, the second moment of the line profile, measured from high $S / N\left(\gtrsim 10\right.$ pixel $\left.^{-1}\right)$ data, as a means to characterize the BLR gas velocity. In this case, Denney et al. [46] find that the scatter in $\sigma_{l}$-based CIV masses compared with $\mathrm{H} \beta$ masses is $<0.3$ dex, as shown in Figure 8.

Because the strength of the bias seems to be inversely correlated with the FWHM of the CIV line, FWHM-based CIV single-epoch mass scaling relationships can also be calibrated to account for a large part of this bias, leading to a correctable FWHM-based mass [e.g., 47; 38]. These types of additional calibrations are at least more applicable to survey quality data, which typically do not have high enough $S / N$ to reliably measure the line dispersion. This is therefore an acceptable correction until better methods become available. Note, however, that such corrections are sample dependent, and the sample of data available for these types of calibration needs to be increased.

Finally, it is interesting to note that RM time delay measurements are no longer the bottle-neck to increasing the accuracy or precision of AGN BH mass measurements. This is because significant effort has been put into RM campaigns as a means to measure accurate time delays for RM-based masses and to calibrate the $R-L$ relationship for its application of estimating single-epoch masses, which has resulted not only in the consistency of the $R-L$ relationship with physical expectations, but also in a surprisingly small observed scatter. Instead, it is accurately characterizing the relevant, intrinsic velocity field - that associated with the reverberating gas — from the data that remains 


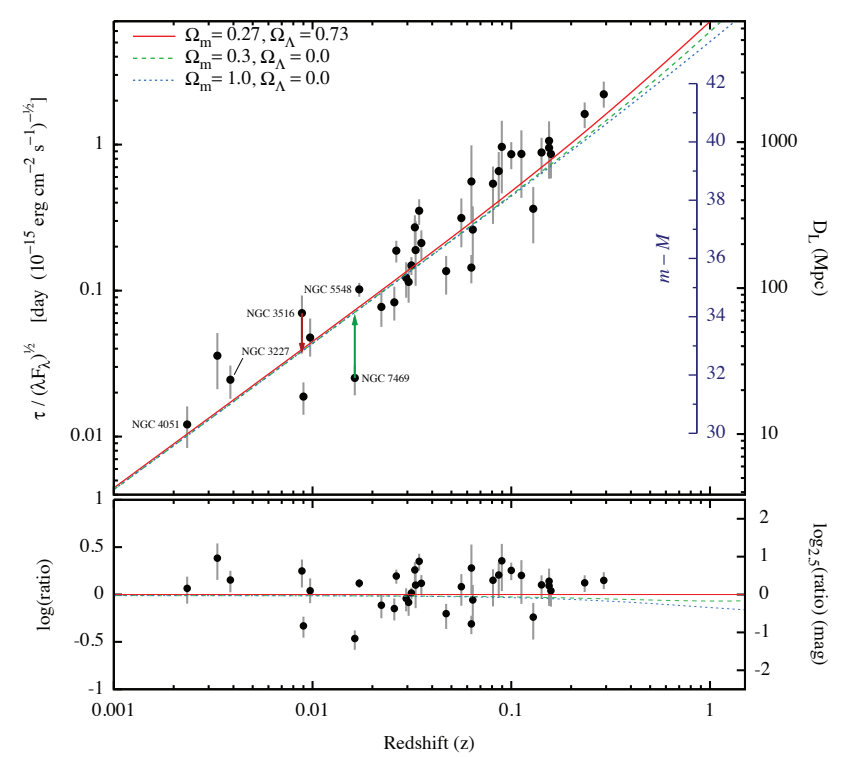

Figure 9: The AGN Hubble diagram, reproduced from Watson et al. [48]; see that work for details. The AGN luminosity distance indicator is relative to the ratio of the reverberation time delay to the observed AGN continuum flux and is shown here as a function of redshift for the sample of 38 AGN considered by Watson et al. The absolute calibration for the luminosity distance and distance modulus $(m-M)$ zero point used here is based on the surface brightness fluctuations distance to NGC 3227. The colored curves are the expected distance - redshift relations for various cosmologies, which agree well with, but are not fit to the data. The bottom panel shows residuals with respect to a $\Lambda \mathrm{CDM}$ universe.

a challenge. Information can be gleaned from the detailed RM analyses described above, but in general, continuing to investigate the best ways to mitigate velocity-dependent biases in $\mathrm{BH}$ masses and to improve methods and models for inferring the intrinsic BLR velocity dispersion from the observable data remains a current topic of research.

\section{AGN Distances Measured from Reverberation Mapping Measurements}

As mentioned above, the most recent, empirical host-starlight-corrected version of the $R-$ $L$ relationship [37] shown in Figure 5 is consistent with the physical expectation that $R \sim L^{0.5}$. Because this empirical relationship holds on physical grounds and exhibits such a small scatter ( $\sim 0.13$ dex), it was recognized that the $R-L$ relationship can be inverted to infer a luminosity distance from the observed AGN flux and measured reverberation time delay [48]. AGN can thus be used, through reverberation mapping analyses, as standard candles and cosmic distance indicators. Such measurements can be used to produce a Hubble diagram for AGN, as shown in Figure 9.

Additional work to understand the potential impact of this new method in constraining cosmological parameters has recently been completed by King et al. [49] and shows that AGNs have the potential to be powerful probes of dark energy. AGN have the benefit of being nearly homogeneously accessible as a cosmological probe across the range $0<z \lesssim 4$, which cannot be said 
for any other single probe. The current estimated scatter in this method is larger than other dark energy probes, such as Type Ia supernovae ( $\mathrm{SNe}$ ) and baryon acoustic oscillations (BAO), but because AGN span this range continuously, they have the unique ability to provide independent consistency checks between these and other methods, for which is there is not yet another single overlapping method. King et al. [49] conducted simulations to estimate the additional constraints AGN distances can place on common cosmological parameters of interest, such as $\Omega_{\mathrm{M}}$ and the dark energy equation of state parameters, $w_{0}$ and $w_{z}$. The constraints resulting from these simulations are shown in Figure 10, based on 2000 AGN distance measurements, or more generally any highredshift standard candle (HzSC), with a sample scatter of 0.2 mag. It is clear from the left panels that significant improvements can be made over the current constraints provided by $\mathrm{SNe}, \mathrm{BAO}$, and the cosmic microwave background (CMB). The improved constraining power of adding $\mathrm{HzSC}$ measurements to currently or soon to be underway "Stage III" (such as DES or Pan-STARRS4) or future "Stage IV" (such as LSST, WFIRST, and Euclid) dark energy experiments is weaker. However, it is important to consider that this is assuming an input, fiducial $\Lambda$ CDM universe, and therefore, improved constraints are weighted toward the importance of information at relatively lower redshifts and do not consider the possibility for exotic changes at high redshift, where a HzSC may have the larger advantage.

There is much observational work yet to be done to optimize reverberation mapping programs for this type of application. For comparison, the current sample, collected for an historically different purpose, largely includes only nearby, apparently bright AGNs, thereby suffering from sources of systematics such as volume-limited selection effects, reddening, and significant host galaxy contamination. While the latter has been corrected through the work of Bentz et al. [37, and references therein], this correction is not perfect, and as such, still forms the source of, in particular, redshiftdependent systematic bias. Future reverberation campaigns performed with the goal of investigating dark energy can mitigate many of the systematics from which the current sample suffers [see 48, for additional discussion]. Work is underway (e.g., Kilerci-Eser, in prep.) to identify sources of scatter in the $R-L$ relationship, to determine how much may be systematic and how much is statistical and therefore able to be reduced with larger samples, and to investigate the best methods for mitigating such sources in future reverberation mapping programs. Such work is important as a means to maximize not only the application of these programs to dark energy studies, but also to BH mass, AGN physics, and galaxy evolution studies.

\section{Summary and Future Prospects}

The practice of reverberation mapping has a history of about 30 years, yet in that span, RM time delays have been measured in only on order 50 sources (albeit some sources have multiple measurements). Traditionally, these observations are obtained through long observing campaigns using long-slit spectrographs on medium aperture (1-2-m class, typically private consortium operated) telescopes. All of the results I have described here have come from this type of reverberation mapping campaign. Significant gains in sample size have therefore been relatively slow; however, with those gains, have come huge leaps in our understanding of AGN physics, the possible applications of the measurements, and broad reaching insight for hunting the 'big game' of astronomy. This was demonstrated here by the discussion (1) of the significant improvements in estimates of 

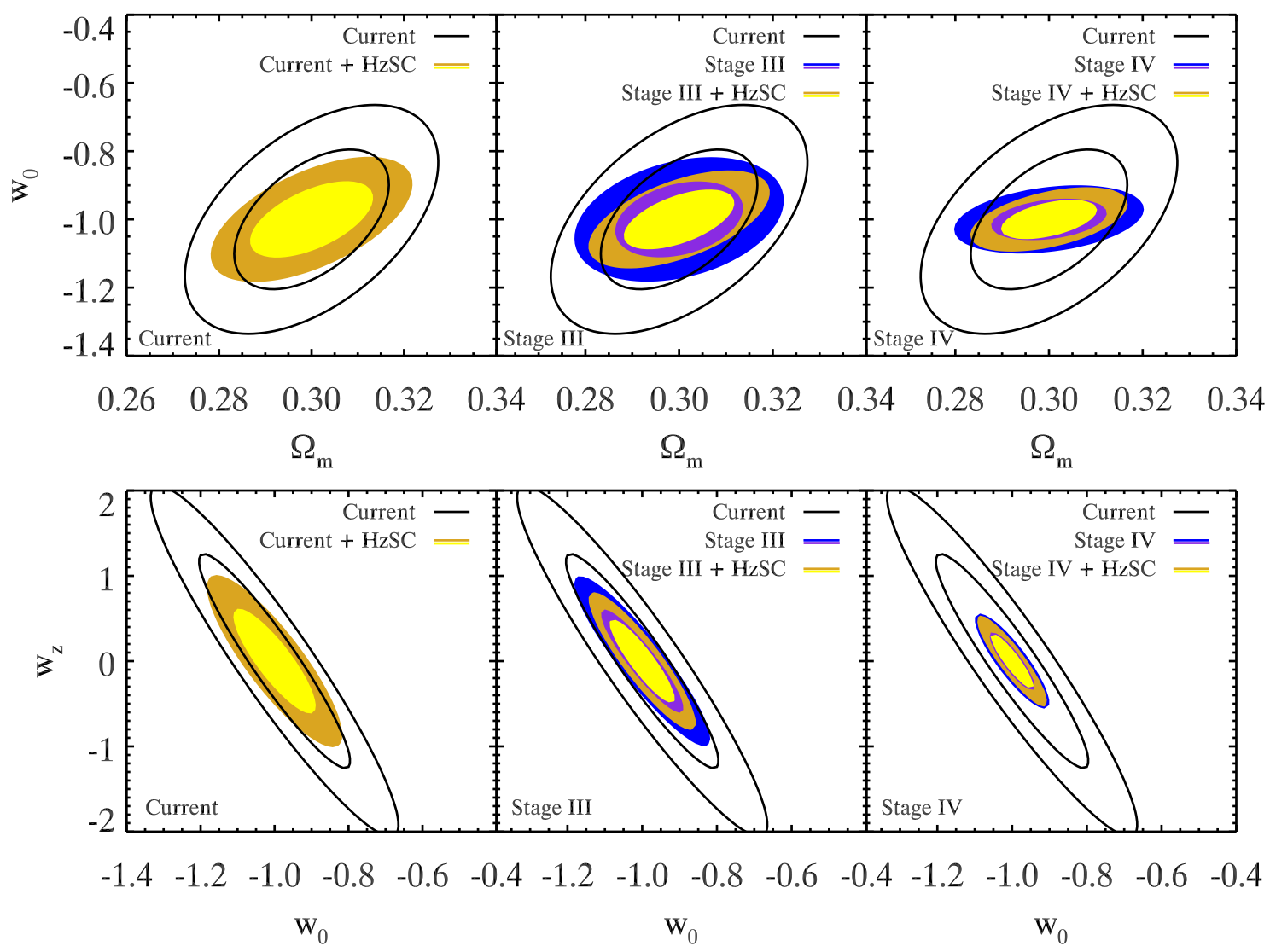

Figure 10: The 1- and 2- sigma level confidence contours, showing the constraints on combinations of the dark energy-related cosmological parameters $\Omega_{m}, w_{0}$, and $w_{z}$. The black contours show the current Type Ia $\mathrm{SNe}, \mathrm{BAO}$, and CMB constraints only, the blue/purple contours show the predicted future constraints from Stage III (middle) and Stage IV (right) SNe, BAO and CMB data, and the orange/yellow contours in each panel combine those constraints with a high-redshift standard candle, such as AGN. From King et al. [49]; see that work for details.

cosmologically distant black hole masses, which, when is comes down to it, are basically all dependent on reverberation mapping measurements, (2) of the ability of reverberation mapping to probe size scales unresolvable by even the largest telescopes currently being conceived, by exploiting time-domain astrophysics, and (3) of the wide spread application of reverberation mapping results, from understanding the details of photoionization and line emission at the atomic level, to probing cosmic distances and the expansion history of the universe.

While significant and exciting advancements in reverberation mapping have come in the last 10 years, the next 10 hold the promise to be even more exciting, thanks to new technology, innovations in RM campaign observing strategies, and expanded interest in this field (likely because of the current, or potential, applications of this technique). Such innovations have created the possibility for our current reverberation mapping sample of AGN to increase by a factor of 10, or even 100, and the redshift range probed to extend to $z \sim 3$ or beyond. These advances will require a number of complementary new approaches (compared to previous methods). These include: 
- Large reverberation mapping "surveys" will use wide-field multi-object spectrographs like the BOSS spectrograph on the SDSS telescope (PI Y. Shen and W.N. Brandt, as an ancillary program of SDSS-III), and the AAOmega spectrograph on the Anglo-Australian telescope (PI P. Martini, as an ancillary program of DES) to significantly increase the sample sizes.

- Reverberation mapping of lensed quasars on innovative instruments such as the GMOS or NIFS integral field spectrographs on Gemini and the Xshooter spectrograph on the VLT (PI K. Denney) will increase the efficiency with which we can (i) measure high-redshift time delays, (i) probe the complete low- and high-ionization regions of the BLR, and (iii) mitigate systematics in the data analysis, such as due to slit losses.

- Reverberation mapping programs carried out on robotic networks of telescopes like the LCOGT network (PI D. Sand) will significantly reduce the man-power needed to acquire the data and can conceivably provide the best sampled light curves, i.e., with intra-day cadence, as this network spans the globe.

- Photometric reverberation mapping programs [see, e.g., 50], and/or photometric triggering of spectroscopic observations once continuum variability signals have been identified, could significantly increase the number of objects with measured time delays across a wide range of redshifts in the upcoming LSST era.

- Finally, traditional reverberation mapping programs have focused on very high quality data of a small sample of objects to maximize the detailed information that can be learned about individual sources, including precision black hole masses, BLR geometry and kinematics, accretion rates, etc. Yet, the implied amount of data in these future programs moves this field to a whole new statistical level, where the potential exists for stacking analyses of a small amount of data for a large number of sources to provide broader statistics of black hole populations [see 51]. Such analyses will provide complementary information to the detailed studies of individual objects to help expand our view of the role of BHs and AGN on their environments and over cosmic time.

\section{References}

[1] M. Colless et al., The 2dF Galaxy Redshift Survey: spectra and redshifts, MNRAS 328 (Dec., 2001) 1039-1063, [astro-ph/0106498].

[2] C. Stoughton et al., Sloan Digital Sky Survey: Early Data Release, AJ 123 (Jan., 2002) $485-548$.

[3] J. R. Bond, L. Kofman, and D. Pogosyan, How filaments of galaxies are woven into the cosmic web, Nature 380 (Apr., 1996) 603-606, [astro-ph/9512141].

[4] V. Springel et al., Simulations of the formation, evolution and clustering of galaxies and quasars, Nature 435 (June, 2005) 629-636, [astro-ph / 0504097 ].

[5] L. Ferrarese and D. Merritt, A Fundamental Relation between Supermassive Black Holes and Their Host Galaxies, ApJL 539 (Aug., 2000) L9-L12. 
[6] K. Gebhardt et al., A Relationship between Nuclear Black Hole Mass and Galaxy Velocity Dispersion, ApJL 539 (Aug., 2000) L13-L16.

[7] J. Magorrian et al., The Demography of Massive Dark Objects in Galaxy Centers, AJ 115 (June, 1998) 2285-2305, [arXiv: astro-ph/9708072].

[8] A. W. Graham, The black hole mass - spheroid luminosity relation, MNRAS 379 (Aug., 2007) 711-722, [0 705.0618].

[9] M. C. Bentz, B. M. Peterson, R. W. Pogge, and M. Vestergaard, The Black Hole Mass-Bulge Luminosity Relationship for Active Galactic Nuclei From Reverberation Mapping and Hubble Space Telescope Imaging, ApJL 694 (Apr., 2009) L166-L170, [0 812 . 228 4].

[10] K. Jahnke and A. V. Macciò, The Non-causal Origin of the Black-hole-galaxy Scaling Relations, ApJ 734 (June, 2011) 92, [1006.0482].

[11] N. J. McConnell and C.-P. Ma, Revisiting the Scaling Relations of Black Hole Masses and Host Galaxy Properties, ApJ 764 (Feb., 2013) 184, [1211.2816].

[12] C. J. Grier et al., Stellar Velocity Dispersion Measurements in High-Luminosity Quasar Hosts and Implications for the AGN Black Hole Mass Scale, ApJ 773 (Aug., 2013) 90, [1305.2447].

[13] J. R. Herrnstein, J. M. Moran, L. J. Greenhill, and A. S. Trotter, The Geometry of and Mass Accretion Rate through the Maser Accretion Disk in NGC 4258, ApJ 629 (Aug., 2005) 719-738, [astro-ph/0504405].

[14] A. L. Argon, L. J. Greenhill, M. J. Reid, J. M. Moran, and E. M. L. Humphreys, Toward a New Geometric Distance to the Active Galaxy NGC 4258. I. VLBI Monitoring of Water Maser Emission, ApJ 659 (Apr., 2007) 1040-1062, [astro-ph / 0701396 ].

[15] J. Kormendy and L. C. Ho, Coevolution (Or Not) of Supermassive Black Holes and Host Galaxies, ARA\&A 51 (Aug., 2013) 511-653, [1304 . 7762].

[16] R. D. Blandford and C. F. McKee, Reverberation mapping of the emission line regions of Seyfert galaxies and quasars, ApJ 255 (Apr., 1982) 419-439.

[17] B. M. Peterson, Reverberation mapping of active galactic nuclei, PASP 105 (Mar., 1993) 247-268.

[18] C. A. Onken, L. Ferrarese, D. Merritt, B. M. Peterson, R. W. Pogge, M. Vestergaard, and A. Wandel, Supermassive Black Holes in Active Galactic Nuclei. II. Calibration of the Black Hole Mass-Velocity Dispersion Relationship for Active Galactic Nuclei, ApJ 615 (Nov., 2004) 645-651.

[19] D. Park, B. C. Kelly, J.-H. Woo, and T. Treu, Recalibration of the Virial Factor and $M_{B H^{-}} \sigma_{*}$ Relation for Local Active Galaxies, ApJS 203 (Nov., 2012) 6, [1209. 3773]. 
[20] A. W. Graham, C. A. Onken, E. Athanassoula, and F. Combes, An expanded $M_{b h}-\sigma$ diagram, and a new calibration of active galactic nuclei masses, MNRAS 412 (Apr., 2011) 2211-2228, [1007.3834].

[21] M. C. Bentz et al., The Lick AGN Monitoring Project: Broad Line Region Radii and black Hole Masses from Reverberation Mapping of H $\beta$, ApJ 705 (Nov., 2009) 199-217, [0908.0003].

[22] K. D. Denney et al., Reverberation Mapping Measurements of Black Hole Masses in Six Local Seyfert Galaxies, ApJ 721 (Sept., 2010) 715-737, [1006.4160].

[23] C. J. Grier et al., Reverberation Mapping Results for Five Seyfert 1 Galaxies, ApJ 755 (Aug., 2012) 60, [1206.6523].

[24] A. J. Barth et al., The Lick AGN Monitoring Project 2011: Fe II Reverberation from the Outer Broad-line Region, ApJ 769 (June, 2013) 128, [1304 . 4643].

[25] C. J. Grier et al., The Structure of the Broad-line Region in Active Galactic Nuclei. I. Reconstructed Velocity-delay Maps, ApJ 764 (Feb., 2013) 47, [1210.2397].

[26] A. Pancoast $e$ al., The Lick AGN Monitoring Project 2011: Dynamical Modeling of the Broad-line Region in Mrk 50, ApJ 754 (July, 2012) 49, [1205. 3789 ].

[27] B. J. Brewer et al., The Mass of the Black Hole in Arp 151 from Bayesian Modeling of Reverberation Mapping Data, ApJL 733 (June, 2011) L33, [1104 . 4794].

[28] A. Pancoast, B. J. Brewer, T. Treu, D. Park, A. J. Barth, M. C. Bentz, and J.-H. Woo, Modeling reverberation mapping data II: dynamical modeling of the Lick AGN Monitoring Project 2008 dataset, submitted to MNRAS (Nov., 2013), [1311. 6475].

[29] M. C. Bentz et al., NGC 5548 in a Low-Luminosity State: Implications for the Broad-Line Region, ApJ 662 (June, 2007) 205-212, [arXiv: astro-ph / 0702644 ].

[30] K. Davidson, Photoionization and the Emission-Line Spectra of Quasi-Stellar Objects, ApJ 171 (Feb., 1972) 213.

[31] J. H. Krolik and C. F. McKee, Hydrogen emission-line spectra in quasars and active galactic nuclei, ApJS 37 (Aug., 1978) 459-483.

[32] A. P. Koratkar and C. M. Gaskell, Radius-luminosity and mass-luminosity relationships for active galactic nuclei, ApJL 370 (Apr., 1991) L61-L64.

[33] S. Kaspi, P. S. Smith, H. Netzer, D. Maoz, B. T. Jannuzi, and U. Giveon, Reverberation Measurements for 17 Quasars and the Size-Mass-Luminosity Relations in Active Galactic Nuclei, ApJ 533 (Apr., 2000) 631-649.

[34] S. Kaspi, D. Maoz, H. Netzer, B. M. Peterson, M. Vestergaard, and B. T. Jannuzi, The Relationship between Luminosity and Broad-Line Region Size in Active Galactic Nuclei, ApJ 629 (Aug., 2005) 61-71. 
[35] M. C. Bentz, B. M. Peterson, R. W. Pogge, M. Vestergaard, and C. A. Onken, The Radius-Luminosity Relationship for Active Galactic Nuclei: The Effect of Host-Galaxy Starlight On Luminosity Measurements, ApJ 644 (Feb., 2006) 133.

[36] M. C. Bentz, B. M. Peterson, H. Netzer, R. W. Pogge, and M. Vestergaard, The Radius-Luminosity Relationship for Active Galactic Nuclei: The Effect of Host-Galaxy Starlight on Luminosity Measurements. II. The Full Sample of Reverberation-Mapped AGNs, ApJ 697 (May, 2009) 160-181, [0 812.2283].

[37] M. C. Bentz et al., The Low-Luminosity End of the Radius-Luminosity Relationship for Active Galactic Nuclei, ApJ 767 (Apr., 2013) 149, [1303.1742].

[38] D. Park, J.-H. Woo, K. D. Denney, and J. Shin, Calibrating C-IV-based Black Hole Mass Estimators, ApJ 770 (June, 2013) 87, [1304 . 7281].

[39] J. Wang, X. Dong, T. Wang, L. C. Ho, W. Yuan, H. Wang, K. Zhang, S. Zhang, and H. Zhou, Estimating Black Hole Masses in Active Galactic Nuclei Using the Mg II $\lambda 2800$ Emission Line, ApJ 707 (Dec., 2009) 1334-1346, [0910 .2848].

[40] A. Rafiee and P. B. Hall, Biases in the quasar mass-luminosity plane, MNRAS 415 (Aug., 2011) 2932-2941, [1011.1268].

[41] K. D. Denney, Are Outflows Biasing Single-Epoch CIV Black Hole Mass Estimates?, ApJ 759 (Oct., 2012) 44, [1208.3465].

[42] Y. Shen et al., A Catalog of Quasar Properties from Sloan Digital Sky Survey Data Release 7, ApJS 194 (June, 2011) 45, [1006.5178].

[43] A. Baskin and A. Laor, What controls the CIV line profile in active galactic nuclei?, MNRAS 356 (Jan., 2005) 1029-1044, [arXiv:astro-ph/ 0409196$].$

[44] H. Netzer, P. Lira, B. Trakhtenbrot, O. Shemmer, and I. Cury, Black Hole Mass and Growth Rate at High Redshift, ApJ 671 (Dec., 2007) 1256-1263, [0 088 . 3787].

[45] Y. Shen and X. Liu, Comparing Single-epoch Virial Black Hole Mass Estimators for Luminous Quasars, ApJ 753 (July, 2012) 125, [1203.0601].

[46] K. D. Denney, R. W. Pogge, R. J. Assef, C. S. Kochanek, B. M. Peterson, and M. Vestergaard, C IV Line-width Anomalies: The Perils of Low Signal-to-noise Spectra, ApJ 775 (Sept., 2013) 60, [1303.3889].

[47] J. C. Runnoe, M. S. Brotherton, Z. Shang, and M. A. DiPompeo, Rehabilitating CIV-based black hole mass estimates in quasars, MNRAS 434 (Sep, 2013) 848-861, [1306 . 3521].

[48] D. Watson, K. D. Denney, M. Vestergaard, and T. M. Davis, A new cosmological distance measure using AGN, ApJL $\mathbf{7 4 0}$ (Oct., 2011) L49, [110 9 . 4632]. 
[49] A. L. King, T. M. Davis, K. Denney, M. Vestergaard, and D. Watson, High Redshift Standard Candles I: Predicted Cosmological Constraints, submitted to MNRAS; ArXiv:1311.2356 (Nov., 2013) [1311.2356].

[50] Y. Zu, C. S. Kochanek, S. Kozłowski, and B. M. Peterson, Reverberation Mapping with Photometry, submitted to ApJ ArXiv:1310.6774 (Oct., 2013) [1310.6774].

[51] S. Fine et al., Stacked reverberation mapping, MNRAS 434 (July, 2013) L16-L20. 